\title{
RECEPCIÓN JURISPRUDENCIAL DEL PRINCIPIO DE PROPORCIONALIDAD EN MÉXICO
}

\section{EL PRINCIPIO DE PROPORCIONALIDAD}

El principio de proporcionalidad o razonabilidad, como se sabe, es una de las herramientas metodológicas más importantes del constitucionalismo de nuestro tiempo; permite superar la aplicación de métodos tradicionales, literalistas y estrechos en la interpretación de la ley fundamental, apoyando la solidez de las conclusiones jurisdiccionales - y al menos idealmente también la argumentación de otros operadores jurídicos: legislador, administración pública y postulantes- $-{ }^{1}$ La detallada elaboración de sus diversos subprincipios (idoneidad, necesidad y proporcionalidad stricto sensu) ${ }^{2}$ ha permitido resolver, siquiera al brindarle parámetros objetivos, el problema de dar contenido a la volátil idea de "razonabilidad", tan invocada en todos los ámbitos jurídicos pero más en el constitucional por la indeterminación de sus disposiciones; muy pocos impugnarán que con su empleo "es posible establecer resultados o decisiones de manera racional que son bastante aceptables, lo que justifica totalmente el método". ${ }^{3}$ Pero este principio no constituye una fórmula absoluta para la aplicación jurídica; da criterios para

1 Cfr. Bernal Pulido, Carlos, El principio de proporcionalidad y los derechos fundamentales, 2a. ed., Madrid, CEPC, 2005, pp. 544 y 545.

2 Entendidas como: idoneidad, la aptitud de la medida examinada para lograr el fin que se proponga; necesidad, que sea la menos lesiva al derecho o principio que afecta; y proporcionalidad en sentido estricto, la existencia de un perjuicio a éste menor o igual al beneficio que obtiene el fin perseguido por la medida en cuestión.

3 Cárdenas Gracia, Jaime, La argumentación como derecho, México, UNAM, Instituto de Investigaciones Jurídicas, 2005, p. 150. 
ella y tiene un procedimiento bien establecido, pero no son fines en sí mismos sino que buscan, tendiendo naturalmente a ello, lograr la "igualdad proporcional" entre bienes jurídicos tutelados o tutelables - para el caso de su empleo legislativo-, en la cual precisamente consiste la justicia. ${ }^{4}$

La proporcionalidad no es una idea nueva, ni siquiera para el derecho que con diversas modalidades la usa desde mucho tiempo atrás en varios de sus ámbitos: internacional, penal, civil, etcétera, ${ }^{5}$ de manera que el principio respectivo no impera sólo en el constitucional. Pero ciertamente en éste adquiere gran relevancia por la necesidad de utilizarlo para resolver múltiples problemas, cuya solución sin él sería sencillamente dejada al decisionismo y la arbitrariedad: el control de las amplias facultades discrecionales que da la Constitución (sobre todo al legislador, pero también a la administración y la judicatura), la aplicación del principio de igualdad (y la relevancia de las diferencias para la razonabilidad de una distinción legislativa); ${ }^{6}$ el contenido esencial de los derechos fundamentales y los límites de sus posibles restricciones (sobre todo cuando las cláusulas generales constitucionales dejan establecerlas a los "términos que indique la ley"); y last but not least, los "conflictos" de los derechos fundamentales entre sí y con otros principios constitucionales -e incluso entre reglas derivadas de un solo y mismo principio-.

4 Recaséns Siches, Luis, Tratado general de filosofía del derecho, 14a. ed., México, Porrúa, 1999, p. 481.

5 Matscher, Franz, "Les contraintes de l'interprétation juridictionelle. Les méthodes d'interprétation de la Convention Européenne", en Sudre, Frédéric (dir.), L'interprétation de la Convention Européenne des Droits de l'Homme, Bruselas, Nemesis-Bruylant, 1998, p. 37.

6 La aplicación del principio de proporcionalidad a este tema es de lo más complicada, debido a la construcción —inspiradas en la "heightened equal protection" norteamericana - de diferentes "intensidades" del examen judicial, según la distinción de trato analizada incida o no en derechos fundamentales o prohibiciones constitucionales expresas de discriminación. Véase "IGUALDAD. CASOS EN LOS QUE EL JUEZ CONSTITUCIONAL DEBE HACER UN ESCRUTINIO ESTRICTO DE LAS CLASIFICACIONES LEGISLATIVAS (INTERPRETACIÓN DEL ARTíCULO 10. DE LA CONSTITUCIÓN POLÍTICA DE LOS ESTADOS UNIDOS MeXICANOS)", tesis 1a./J. 37/2008, Semanario Judicial de la Federación y su Gace$t a$, novena época, abril de 2008, t. XXVII, p. 175; e "IGUALDAD. CASOS EN LOS QUE EL JUZGADOR CONSTITUCIONAL DEBE ANALIZAR EL RESPETO A DICHA GARANTÍA CON MAYOR INTENSIDAD", tesis 2a. LXXXV/2008, Semanario Judicial de la Federación, novena época, junio de 2008, t. XXVII, p. 439. 
El principio de proporcionalidad es de carácter estructural del ordenamiento jurídico, como el de no contradicción y el de exclusión (lo que no está prohibido, está permitido), que constituyen "reglas de reconocimiento" para la pertenencia de una norma al sistema; ${ }^{7}$ quizá por tal motivo se le atribuyó un carácter jusnaturalista ${ }^{8}$ que nunca vio con buenos ojos el formalismo al que estamos acostumbrados. No hay salida: si queremos un ordenamiento "razonable" y materialmente "correcto", sus normas — todas y cada una - deberán cumplir dicho principio y ser idóneas, necesarias y proporcionadas a un fin legítimo; se trata de un "orden natural de las cosas" que no debe preterirse ni puede suprimirse.

Como veremos en la siguiente sección, nuestro objeto general de estudio - el específico es su pleno reconocimiento jurisprudencial en nuestro país - se ha establecido en las jurisdicciones nacionales e internacionales más diversas, con diferentes nombres y modalidades, con énfasis en todos o sólo alguno(s) de sus aspectos, pero concurriendo en términos generales a lo mismo. México no participaba claramente en este concierto internacional hacia la razonabilidad ${ }^{9}$ del orden jurídico; aunque su jurisprudencia del siglo XX dio algunos pasos en ese camino, éstos siempre fueron insuficientes y hasta apenas hace una década que, con no pocos trabajos, el principio de proporcionalidad fue abriéndose paso en las decisiones de nuestros tribunales. En este curso, la jurisprudencia 130/2007 del Pleno de la Suprema Corte de Justicia de la Nación $(\mathrm{SCJN})^{10}$ marca un hito al establecer, con pleno carácter vinculante para todos los tribunales mexicanos, la obligación de observar dicho principio y darle fundamento textual en el artículo 16 de la Constitución - lo que

7 Véase Atienza, Manuel y Ruiz Manero, Juan, "La regla de reconocimiento", en Laporta, Francisco J. (ed.), Constitución: problemas filosóficos, Madrid, Ministerio de la Presidencia-CEPC, 2003, pp. 131 y 132.

8 Cfr. Hotz, Werner Friedrich, Zur Notwendigkeit und Verhältnismässigkeit von Grundrechtseingriffen, Zurich, Schulthess Polygraphischer, 1977, p. 1.

9 Entendiendo como "razonabilidad" la mejor adecuación entre fines y medios, dando "cuenta de las circunstancias y limitaciones que implican, con la renuncia a una actitud... de absolutismo". Véase Abbagnano, Nicola, Diccionario de filosofía, 4a. ed., actualizada y aumentada por Giovanni Fornero, trad. de José Esteban Calderón et al., México, Fondo de Cultura Económica, 2004, voz: "Razonable".

10 En lo sucesivo, usaremos esta abreviatura para referirnos genéricamente a la SCJN; de hacerlo específicamente, indicaremos sólo los órganos de la misma a que aludiremos: Pleno, Primera y Segunda Salas. 
entre nosotros no es de poca monta-, concretamente en su garantía de motivación material.

\section{REPASO COMPARADO E INTERNACIONAL}

Diversas jurisdicciones han usado este principio con varias denominaciones y caracterizaciones: entendiendo como un conjunto unitario a sus subprincipios (idoneidad, necesidad y proporcionalidad stricto sensu) o como elementos separados y ajenos entre sí, o bien fusionando algunos de sus subprincipios en uno solo (generalmente los de idoneidad y necesidad), o incluso agregándole un "tercer elemento": la licitud de los fines que persiga la medida enjuiciada por este criterio. ${ }^{11}$ Sin embargo, por el detalle de sus elementos que ayuda a un examen matizado y específico, atendemos su formulación clásica y más extendida como un solo principio (proporcionalidad lato sensu) ${ }^{12}$ que se subdivide en los tres subprincipios mencionados, cuya satisfacción concurrente indica la regularidad de un acto estatal porque unidos "expresan un conjunto de condiciones de racionalidad que toda medida estatal debe cumplir". ${ }^{13}$ A pesar de las diferencias hablamos prácticamente de lo mismo al referir la proporcionalidad, la razonabilidad, la idoneidad y la necesidad.

Al tratar este tema es obligatorio acudir primeramente a la doctrina y jurisprudencia alemanas, pues precisamente en ese país se llevó a cabo la elaboración de los tres subprincipios de la proporcionalidad; el mismo Robert Alexy ha dicho que la garantía del "contenido esencial" de los derechos fundamentales a que se refiere el artículo 19.2 de la Constitución germana - y las de otros países como España- es precisamente el cumplimiento del principio de proporcionalidad. ${ }^{14}$ El Tribunal Constitucional Federal alemán definió la proporcionalidad lato sensu como de "rango constitucional" y la derivó "del principio de Estado de derecho y también... de la esencia misma de los derechos fundamentales". ${ }^{15}$

11 Que más bien es un presupuesto del examen de proporcionalidad, un "prius lógico" y no uno de sus elementos, como ha dicho el Tribunal Constitucional español (STC 55/1996, F. J. 7).

12 Bernal Pulido, op. cit., nota 1, p. 38.

13 Ibidem, p. 537.

14 BVerfGE 30, 292 (316 y 317); y Alexy, Robert, Theorie der Grundrechte, 4a. ed., Fráncfort del Meno, Suhrkamp, 2001, pp. 100-104 y 269.

15 BVerfGE 19, 342, pp. 348 y 349. 
Puede decirse que de Alemania tomó el Tribunal Europeo de Derechos Humanos el principio de proporcionalidad, al que ha dado un frecuente uso y enriquecido con el concepto de "margen nacional de apreciación"16 que permite cierta amplitud a los Estados - aunque con diversos alcances, siempre hay tal "margen" en la aplicación del principio de proporcionalidad-. Enjuiciando el respeto al derecho de igualdad, este órgano internacional habló de que las medidas estatales distintivas deben tener una "justificación objetiva y razonable" y una "relación razonable de proporcionalidad entre los medios empleados y el fin perseguido". ${ }^{17}$

Con explícita inspiración en la anterior sentencia del Tribunal de Estrasburgo y haciendo suyos casi los mismos términos, el Tribunal Constitucional Español también adoptó el principio de proporcionalidad; ${ }^{18}$ y también le ha otorgado diversos perfiles elementales como ya vimos, ${ }^{19}$ aportando sus propias observaciones a su concepción genérica. La recepción hispana de este principio es sumamente importante; por la cercanía de nuestra lengua y la formación académica de muchos juristas de estas latitudes, a través de la doctrina y jurisprudencia españolas, los países latinoamericanos han podido hospedar en sus decisiones jurisdiccionales - las mexicanas inclusive - el principio de proporcionalidad.

Igualmente en la jurisprudencia norteamericana encontramos (veladas) aplicaciones de los subprincipios de la proporcionalidad, aunque es mucho más conocido su enfoque del llamado balancing test o "ponderación". ${ }^{20} \mathrm{Al}$ respecto cabe notar que el principio de proporcionalidad abarca el balancing sin agotarse en él, ya que éste equivale sólo a uno de sus

16 Handyside c. Royaume-Uni, núm. 5493/72, fondo, 7 de diciembre de 1976, §§ 47 y 48.

17 Affaire "relative à certains aspects du régime linguistique de l'enseignement en Belgique" c. Belgique, núms. 1474/62 et al., fondo, 23 de julio de 1968, cons. jurs., § B.10. Este pasaje recuerda una de las primeras sentencias alemanas que habló de la regularidad de las distinciones legislativas bajo justificaciones "razonables" y "objetivas", resultantes de "la naturaleza de las cosas": $B \operatorname{VerfGE~1,~14,~p.~} 52$.

18 STC 22/1981, F.J. 3.

19 Supra, nota 2.

20 United States vs. O'Brien, 391 U.S. 367, 377 (1968); y Pickering vs. Board of Education, 391 U.S. 563, 568, 1968. 
subprincipios: el de proporcionalidad stricto sensu o ponderación; ${ }^{21}$ de manera que no deben confundirse.

También Francia ha recurrido al principio de proporcionalidad, aunque con menor frecuencia - dado el parco estilo de su Consejo Constitucional-: este órgano declaró la inconstitucionalidad del requerimiento de una autorización administrativa para el cambio de giro de locales mercantiles de algunas ciudades, por infligir un "perjuicio [al derecho de propiedad y a la libertad de empresa] desproporcionado al objetivo perseguido". 22

Dejándolo para lo último, por su relevancia en Latinoamérica y en particular por la vinculación que México tiene a su jurisprudencia ${ }^{23}$ (entre otros por lo dispuesto en los artículos 31.3 de la Convención de Viena sobre el Derecho de los Tratados, y 62.3 de la Convención Americana sobre Derechos Humanos); recordamos que la Corte Interamericana de Derechos Humanos afirmó que el principio de proporcionalidad es "indispensable" en una "sociedad democrática", y por tanto las afectaciones a un derecho fundamental son lícitas únicamente cuando

...estén orientadas a satisfacer un interés público imperativo. Entre varias opciones para alcanzar ese objetivo, debe escogerse aquella que restrinja en menor escala el derecho protegido. Dado este estándar, no es suficiente que se demuestre, por ejemplo, que la ley cumple un propósito útil u oportuno... la restricción debe ser proporcional al interés que la justifica y ajustarse estrechamente al logro de ese legítimo objetivo, interfiriendo en la menor medida posible en el efectivo ejercicio del derecho [fundamental]. ${ }^{24}$

21 Alexy, Robert, "Constitutional Rights, Balancing, and Rationality", Ratio Juris, Oxford, vol. 16, núm. 2, junio de 2003, p. 135.

22 Decisión 2000-436 DC, §§ 12, 15 y 20.

23 Como acertadamente reconoció el ministro Góngora Pimentel en su voto particular de la acción de inconstitucionalidad 26/2006 (Diario Oficial de la Federación, 20 de agosto de 2007, 3a. sección, pp. 80 y 93). Véase "Decreto Promulgatorio de la Declaración para el Reconocimiento de la Competencia Contenciosa de la Corte Interamericana de Derechos Humanos", ibidem, 24 de febrero de 1999, 1a. sección, p. 2 (aclaración en idem, 25 de febrero de 1999, 1a. sección, p. 7).

24 Ricardo Canese, fondo y reparaciones, 31 de agosto de 2004, serie C, núm. 111, $\S 996$ y 129. 


\section{LAS PRIMERAS TESIS MEXICANAS}

En las primeras épocas de la jurisprudencia mexicana posterior a 1917, ya hubo algunos pronunciamientos de la SCJN para evitar la arbitrariedad y excesos de las autoridades, aun las legislativas, en el uso de sus facultades. ${ }^{25}$ Pero dichos precedentes resultaban insuficientes por indeterminados, dando manga ancha al operador jurídico por usar sin especificación conceptos como "razonable" o prohibir genéricamente las actuaciones "caprichosas" de la autoridad.

Algún avance hubo hasta 1996 cuando el Pleno intentó dar contenido a la garantía de equidad tributaria — que hasta hace unos años prácticamente era lo único sobre lo que versaba la doctrina de igualdad de ese tribunal- hablando de un "juicio de equilibrio en sede constitucional", que debía aprobar una distinción legislativa para satisfacer ese derecho fundamental. ${ }^{26}$ Este criterio en realidad fue la incompleta calca de una importantísima línea jurisprudencial del Tribunal Constitucional español, ${ }^{27}$ que en su asimilación en nuestro sistema perdió mucho de su sentido original, lo cual ocasionó más confusión que certidumbre - y tampoco eliminó la indeterminación del examen de esa garantía-.

No expresamente y con timidez, la SCJN desde entonces aplicó el principio de proporcionalidad, o lo sugería para el analizar la validez de restricciones a los derechos fundamentales, pero sin la claridad que se hubiera querido. Son ejemplos notables los casos relativos al acceso a la

25 "FACULTADES DISCRECIONALES. APRECIACIÓN DEL USO INDEBIDO DE ELLAS EN EL JUICIO DE AMPARO", tesis 38, Apéndice al Semanario Judicial de la Federación 1917-2000, t. I, p. 45; y "LIBERTAD DE TRABAJO", Semanario Judicial de la Federación, quinta época, t. LXI, p. 4025.

26 "EQuidAD TRIBUTARIA. SuS ELEMENTOS", tesis 198, Apéndice al Semanario Judicial de la Federación 1917-2000, t. I, p. 240.

27 Por todas, véase STC 76/1990, F.J. 9, inciso $A$ : “[P]ara que la diferenciación resulte constitucionalmente lícita no basta con que lo sea el fin que con ella se persigue, sino que es indispensable además que las consecuencias jurídicas que resultan de tal distinción sean adecuadas y proporcionadas a dicho fin, de manera que la relación entre la medida adoptada, el resultado que se produce y el fin pretendido por el legislador superen un juicio de proporcionalidad en sede constitucional, evitando resultados especialmente gravosos o desmedidos" (cursivas añadidas). 
justicia, la proporcionalidad de las penas y las restricciones a la libertad de trabajo. ${ }^{28}$

De gran importancia en el tema es el criterio de la Sala Superior del Tribunal Electoral del Poder Judicial de la Federación, que data de 2002, por ser el primero que ve panorámicamente el principio de proporcionalidad y compuesto por sus tres mencionados subprincipios - aunque considerándolos separada y no unitariamente-; su relevancia no radica solamente en lo anterior, sino principalmente en haberle dado a dicho principio fundamento textual en los artículos 14 y 16 constitucionales y comenzar a hablar de un "principio de prohibición de excesos o abusos en el ejercicio de facultades discrecionales". ${ }^{29}$

Tampoco puede olvidarse, del Cuarto Tribunal Colegiado en Materia Administrativa, el que lleva el principio de proporcionalidad al derecho procesal constitucional — una aplicación no sustantiva sino adjetiva-, para analizar si procede o no otorgar la suspensión en el juicio de amparo. $^{30}$

\section{ETAPA DE TRANSICIÓN}

A partir de los anteriores pronunciamientos, con mayor frecuencia hallamos en la jurisprudencia mexicana alusiones al principio de proporcionalidad. En el lapso de tiempo al que ahora nos referimos, pueden encon-

28 “GARANTÍA A LA TUTELA JURISDICCIONAL PREVISTA EN EL ARTÍCULO 17 DE LA CONSTitución Política de los Estados Unidos MeXicAnos. Sus AlCANCES”, tesis 1a./J. 42/2007, Semanario Judicial de la Federación y su Gaceta, novena época, t. XXV, abril de 2007, p. 124; y "PRISIÓN VITALICIA. CONSTITUYE UNA PENA INUSITADA DE LAS PROHIBIDAS POR EL ARTÍCULO 22 CONSTITUCIONAL", tesis P./J. 127/2001, Semanario Judicial de la Federación y su Gaceta, novena época, t. XIV, octubre de 2001, p. 15 - aunque luego se revirtió este criterio-y "LIBERTAD DE TRABAJO. NO ES ABSOLUTA DE ACUERDO CON LOS PRINCIPIOS FUNDAMENTALES QUE LA RIGEN (ARTÍCULO 5o., PÁRRAFO PRIMERO, DE LA CONSTITUCIÓN POLÍTICA DE LOS ESTADOS UNIDOS MeXiCANOS)", tesis 162, Apéndice al Semanario Judicial de la Federación 19172000, t. I, p. 143.

29 "PROCEDIMIENTO ADMINISTRATIVO SANCIONADOR ELECTORAL. DEBE REALIZARSE CONFORME A LOS CRITERIOS DE IDONEIDAD, NECESIDAD Y PROPORCIONALIDAD", tesis S3ELJ 62/2002, Compilación Oficial de Jurisprudencia y Tesis Relevantes 1997-2005, p. 235.

30 "TEORÍA DE LOS PRINCIPIOS. SuS ELEMENTOS", tesis I.4o.A.60 K, Semanario Judicial de la Federación y su Gaceta, novena época, t. XXII, septiembre de 2005, p. 1579. 
trarse criterios inspirados por los subprincipios de idoneidad, ${ }^{31}$ necesidad $^{32}$ y proporcionalidad stricto sensu o ponderación; ${ }^{33}$ que son elaborados cada vez con mejor técnica.

De suma importancia son los criterios que la jurisprudencia de la $\mathrm{SCJN}$ estableció en lo que respecta al principio genérico de igualdad y su manifestación concreta en la equidad tributaria. El criterio inicial de esta importante línea, emitido por la Primera Sala, bien puede tenerse como fundacional de la recepción del principio de proporcionalidad en México: en él dicho órgano se ocupa por primera vez de explicar a los tribunales y justiciables la obligatoriedad de calificar tales medidas legislativas de acuerdo con dicho principio, y exponer detalladamente en qué consisten los criterios que lo integran. ${ }^{34}$ Para estudiar la satisfacción del referido principio de justicia fiscal —ámbito natural de su competencia-, la Segunda Sala hizo suyo el anterior criterio y lo perfeccionó para dejar

31 Por ejemplo: "RENTA. El ARTículo 31, FRACCIÓN XII, DE LA LEY DEL IMPUESTO RELATIVO, ES INCONSTITUCIONAL AL LIMITAR LA DEDUCCIÓN DE LOS GASTOS DE PREVISIÓN SOCIAL (LEGISLACIÓN VIGENTE A PARTIR DEL 1o. DE ENERO DE 2003)", tesis P./J. 128/2006, Semanario Judicial de la Federación y su Gaceta, novena época, t. XXIV, noviembre de 2006, p. 7.

32 Asimismo: “Trasplante DE ÓRganos ENTRE VIVOS. El artículo 333, FRACCIÓN VI, DE LA LEY GENERAL DE SALUD, QUE LO PERMITE ÚNICAMENTE ENTRE PERSONAS RELACIONADAS POR PARENTESCO, MATRIMONIO O CONCUBINATO, TRANSGREDE LOS DERECHOS A LA SALUD Y A LA VIDA CONSAGRADOS EN EL ARTíCULO 4o. DE LA CONSTITUCiÓn FEDERAL", tesis P. IX/2003, Semanario Judicial de la Federación y su Gaceta, novena época, t. XVIII, agosto de 2003, p. 54.

33 Véase asimismo: "DERECHOS CONSTITUCIONALES. LA VINCULACIÓN DE SUS LÍMITES EN EL ANÁLISIS DE LA CONSTITUCIONALIDAD DE UNA NORMA SECUNDARIA”, Primer Tribunal Colegiado en Materia Administrativa del Primer Circuito, tesis I.1o.A.100 A, Semanario Judicial de la Federación y su Gaceta, novena época,, t. XVIII, noviembre de 2003, p. 955; “DAÑO MORAL Y DERECHO A LA INFORMACIÓN”, Cuarto Tribunal Colegiado en Materia Civil del Primer Circuito, tesis I.4o.C.57 C, Semanario Judicial de la Federación y su Gaceta, novena época, t. XVII, marzo de 2003, p. 1709 — siguiendo una muy influyente línea española (STC 171/1990, FF.JJ. 4-5)—; y en el mismo derrotero: "LIBERTAD DE EXPRESIÓN E IMPRENTA. LAS LIMITACIONES ESTABLECIDAS POR EL LEGISLADOR RELACIONADAS CON LA VERACIDAD Y CLARIDAD DE LA PUBLICIDAD COMERCIAL SON CONSTITUCIONALES CUANDO INCIDAN EN SU DIMENSIÓN PURAMENTE INFORMATIVA", tesis 1a. CLXV/2004, Semanario Judicial de la Federación y su Gaceta, novena época, t. XXI, enero de 2005, p. 421.

34 "Igualdad. CRiterios para determinar Si El LEgislador RESPETA ESE PRINCIPIO CONSTITUCIONAL", tesis 1a./J. 55/2006, Semanario Judicial de la Federación y su Gaceta, novena época, t. XXIV, septiembre de 2006, p. 75. 
muy claro que la aplicación del principio de proporcionalidad lato sensu precisa de la valoración de determinadas condiciones, de manera escalonada, generando que el incumplimiento de cualquiera de éstas sea suficiente para estimar que existe una violación al indicado principio constitucional, haciendo innecesario el estudio de las demás. ${ }^{35}$

Por lo menos para el examen de distinciones legislativas, en general o en materia tributaria, con los anteriores criterios quedó sentado que el "juicio de equilibrio" a través del cual se debía analizar el cumplimiento de la garantía de igualdad, no es otro sino la aplicación del examen de proporcionalidad. ${ }^{36}$ Esta precisión la reiteró el Pleno en la opinión jurisprudencial que estableció al resolver el importante caso Ley de Medios, sentando que usar dicho principio resulta "necesario" para calificar las diferencias de trato y clasificaciones que haga el legislador. ${ }^{37}$

Sin embargo, en este periodo no se aplicaron con constancia los parámetros del examen de proporcionalidad. El Pleno y las salas en ocasiones lo hicieron equivocada y precariamente: se dijo que era suficiente a una medida legislativa perseguir un fin legítimo para cumplir con ellos, lo cual ya vimos que no es así, pues tal legitimidad es apenas un presupuesto del principio de proporcionalidad. ${ }^{38}$ Igualmente, a pesar de su referido precedente, la Primera Sala opinó que buscar un objetivo legítimo —en tal caso, la imparcialidad de los árbitros en materia de derechos de au-

35 "EQUIDAD TRIBUTARIA. CRITERIOS PARA DETERMINAR SI EL LEGISLADOR RESPETA DICHO PRINCIPIO CONSTITUCIONAL", tesis 2a./J. 31/2007, Semanario Judicial de la Federación y su Gaceta, novena época, t. XXV, marzo de 2007, p. 334. Del mismo órgano también véase "IGUALDAD. CRITERIOS QUE DEBEN OBSERVARSE EN EL CONTROL DE LA CONSTITUCIONALIDAD DE NORMAS QUE SE ESTIMAN VIOLATORIAS DE DICHA GARANTÍA”, tesis 2a. LXXXIV/2008, Semanario Judicial de la Federación y su Gaceta, novena época, t. XXVII, junio de 2008, p. 440.

36 Véase supra, nota 27; Carbonell, Miguel, Los derechos fundamentales en México, 2a. ed., México, Porrúa, CNDH, UNAM, 2006, p. 179; Pérez Portilla, Karla, Principio de igualdad: alcances y perspectivas, México, CONAPRED-UNAM, 2005, p. 97.

37 Acción de inconstitucionalidad 26/2006, Diario Oficial de la Federación, 20 de agosto de 2007, 2a. sección, p. 64.

38 "PRODUCCIÓN Y SERVICIOS. LOS FINES QUE JUSTIFICAN LA EXENCIÓN ESTABLECIDA EN EL ARTÍCULO 80., FRACCIÓN I, INCISO $F$, DE LA LEY DEL IMPUESTO ESPECIAL Relativo, SE SUSTENTAN EN EL INTERÉS DE PROTEGER A LA INDUSTRIA AZUCARERA (LEGISLACIÓN VIGENTE EN 2004)", tesis 2a. XXIV/2006, Semanario Judicial de la Federación y su Gaceta, novena época, t. XXIII, marzo de 2006, p. 534. 
tor- bastaba para hacer lícita una restricción a la libertad de trabajo, ${ }^{39}$ sin "ir mucho más allá de la indudable legitimidad constitucional de que una norma sobre ejercicio de la función arbitral persiga [ese fin]", y entrar al sutil y profundo estudio que requiere el principio de proporcionalidad, según el voto particular del ministro Cossío Díaz. ${ }^{40}$ Y también se tuvo la opinión de que el "test de razonabilidad" del referido principio, "no constituye propiamente una garantía o derecho fundamental" de indefectible aplicación sino "un método bajo el cual, si el órgano de control constitucional lo estima pertinente, puede emprender el análisis de un asunto". 41

\section{LA JURISPRUDENCIA 130/2007 DEL PLENO}

Este contexto de indeterminación sobre el concepto que estudiamos permite ver la importancia de la jurisprudencia 130/2007 del Pleno de la SCJN, cuyo texto transcribimos. ${ }^{42}$

De los criterios emitidos por la Suprema Corte de Justicia de la Nación se advierte que el cumplimiento de los principios de razonabilidad y proporcionalidad implica que al fijar el alcance de una garantía individual por parte del legislador debe: a) perseguir una finalidad constitucionalmente legítima; $b$ ) ser adecuada, idónea, apta y susceptible de alcanzar el fin perseguido; c) ser necesaria, es decir, suficiente para lograr dicha finalidad, de tal forma que no implique una carga desmedida, excesiva o injustificada para el gobernado $\mathrm{y}, d$ ) estar justificada en razones constitucionales. Lo anterior conforme al principio de legalidad, de

39 Primera Sala, amparo en revisión 1110/2005, 7 de diciembre de 2005.

407 de diciembre de 2005 , p. 1 . Esta opinión disidente, con elocuente título, se publicó académicamente: "La intención no basta. Objetivos legislativos y discriminación normativa", Cuestiones Constitucionales. Revista Mexicana de Derecho Constitucional, México, UNAM, Instituto de Investigaciones Jurídicas, núm. 15, julio-diciembre de 2006, pp. 320-330.

41 Segunda Sala, amparo en revisión 143/2007, 13 de junio de 2007.

42 "GARANTÍAS INDIVIDUALES. EL DESARROLLO DE SUS LÍMITES Y LA REGULACIÓN DE SUS POSIBLES CONFLICTOS POR PARTE DEL LEGISLADOR DEBE RESPETAR LOS PRINCIPIOS DE RAZONABILIDAD Y PROPORCIONALIDAD JURÍDICA", Semanario Judicial de la Federación y su Gaceta, novena época, t. XXVI, diciembre de 2007, p. 8 (cursivas añadidas). No está de más recordar que este criterio es de aplicación obligatoria para todos los tribunales mexicanos, incluyendo las Salas de la SCJN, conforme a los artículos 192 de la Ley de Amparo y 235 de la Ley Orgánica del Poder Judicial de la Federación. 
acuerdo con el cual el legislador no puede actuar en exceso de poder ni arbitrariamente en perjuicio de los gobernados.

El primer aspecto llamativo de esta tesis jurisprudencial es la denominación que da la SCJN al principio que nos ocupa, máxime que nunca le había dado tan manifiestamente su nombre generalmente aceptado: "proporcionalidad"; en el caso Ley de Medios ya había mencionado la doble nomenclatura que emplea la jurisprudencia que comentamos, aclarando que ese término es usado por "parte de la doctrina constitucional" y el de "razonabilidad" es de procedencia anglosajona. ${ }^{43}$ Quizá sea conveniente emplear la última denominación para ser más claros al distinguir la proporcionalidad en los sentidos lato y estricto, como de hecho se estila en Argentina - que siempre, por cierto, ha tenido gran influencia norteamericana—;4 y para México lo sea más todavía con el fin de evitar perniciosas confusiones (que sí han ocurrido) con la garantía de "proporcionalidad tributaria", que en nuestro sistema equivale a la "capacidad contributiva" — prácticamente en la totalidad de los casos actuales - y también a un límite (muy indeterminado, pero restrictivo al cabo) a la potestad impositiva del Estado que impide a las contribuciones ser "exorbitantes y ruinosas" para los contribuyentes. ${ }^{45}$

En las ejecutorias que originaron la jurisprudencia que comentamos, ${ }^{46}$ la SCJN reconoció que el principio de proporcionalidad no es novedoso en nuestro medio, pues ya diversos tribunales mexicanos - ella misma entre ellos - se han ocupado de introducir ese concepto, "en orden a racionalizar y hacer transparente el método de resolución de conflictos entre principios constitucionales" 47 y relaciona diversos criterios que ha dictado, algunos muy antiguos, en que alude conjunta o aisladamente a

43 Supra, nota 35.

44 Véase Cianciardo, Juan, El principio de razonabilidad. Del debido proceso sustantivo al moderno juicio de proporcionalidad, Buenos Aires, Ábaco-Universidad Austral, 2004.

45 "IMPUESTOS, EQUIDAD Y PROPORCIONALIDAD DE LOS", tesis 244, Apéndice al Semanario Judicial de la Federación 1917-2000, t. I, p. 290; y "CAPACIDAD CONTRIBUTIVA. CONSISTE EN LA POTENCIALIDAD REAL DE CONTRIBUIR A LOS GASTOS PÚBLICOS”, Semanario Judicial de la Federación y su Gaceta, novena época,t. I, tesis 129, p. 163.

46 Se citará la relativa al amparo en revisión 307/2007, publicada en el Semanario Judicial de la Federación y su Gaceta, paradigma de los demás asuntos que constituyeron la jurisprudencia 130/2007 y que se resolvieron en sus mismos términos (novena época, t. XXVII, abril de 2008, p. 848).

47 Ibidem, pp. 831-837. 
los subprincipios de idoneidad, necesidad y ponderación, algunos de los cuales citamos en este trabajo. El esfuerzo de nuestro tribunal constitucional por dar "abolengo" al principio de proporcionalidad, se explica por el innegable hecho de que esta noción es demasiado elaborada para que sin más fuera aceptada en nuestro medio jurídico tan conservador en ocasiones; era preciso establecer que no se trataba de un recurso empleado por mera ocurrencia o imitación, sino efectivamente de un desarrollo judicial de muchos años de reflexión y probada eficacia - como realmente sucedió fuera y dentro de México-, e incluso arraigado en la época "clásica" de nuestra jurisprudencia.

La primordial función del examen de proporcionalidad es bien definida por la SCJN en la jurisprudencia 130/2007, para el caso que resolvió al emitirla: "fijar el alcance de una garantía individual por parte del legislador". Con esta hipótesis general, se abarcan prácticamente todos los usos que en la práctica jusfundamental puede tener este principio: límites a restricciones legislativas autorizadas, conflictos de intereses constitucionalmente protegidos, así como la licitud de distinciones y clasificaciones legislativas. Dicho principio puede tener muchos más, aun exclusivamente dentro del ámbito constitucional en el cual su empleo no se restringe al campo jusfundamental, pero reconocemos que es difícil dar una definición general a su respecto o una hipótesis de empleo omnicomprensiva; y la SCJN no pasó por alto lo anterior: dedicó una abundante exposición acerca de las implicaciones funcionales del principio de proporcionalidad, estableciendo su íntima relación con la idea de "contenido esencial" en términos generales y no limitado a los derechos fundamentales con los que normalmente se relaciona, ${ }^{48}$ al anotar con acierto en su ejecutoria que ambos conceptos "son relevantes para la solución de conflictos entre bienes constitucionalmente protegidos", sin distinguir la naturaleza de éstos. ${ }^{49}$ Tal pasaje es sumamente trascendente porque finalmente consagra en nuestra jurisprudencia el concepto de "contenido esencial" el cual ya se tuvo por implícito en el ordenamiento mexicano pese a que nuestra Constitución no lo prevé expresamente, ${ }^{50}$ lo que lle-

48 Véase Sánchez Gil, Rubén, El principio de proporcionalidad, México, UNAM, Instituto de Investigaciones Jurídicas, 2007, pp. 111-115.

49 Op. cit., nota 46, pp. 830 y 831.

50 Acción de inconstitucionalidad 2/2004 (voto particular del ministro Góngora Pimentel), Diario Oficial de la Federación, 5 de abril de 2004, 2a. sección, pp. 92-95; y 
vará a desterrar la arraigada práctica en nuestro medio de que el legislador abuse de la potestad que le otorga la Constitución para regular un derecho fundamental $\mathrm{u}$ otra situación jurídica prevista en ella, para — como dijo la SCJN en las ejecutorias analizadas - "sobreponerse axiológica y materialmente" a ellos; por lo mismo no dejamos de lamentar la ausencia de una tesis sistematizada sobre este punto.

Los criterios de este principio igualmente se encuentran claramente establecidos por la $\mathrm{SCJN}$, con sencillez apartada del rigor teórico y un sentido práctico que conviene a su utilización efectiva por la comunidad jurídica. Un punto importante: al exponer en la jurisprudencia 130/2007 el último elemento del análisis de proporcionalidad, la "ponderación", nuestro tribunal constitucional expresa que la medida legislativa examinada debe "estar justificada en razones constitucionales", lo que resulta muy abstracto y puede inducir a confusión; hubiera sido preferible definir este subprincipio en términos más claros y cercanos a la lapidaria fórmula de Bernal Pulido, ${ }^{51}$ que sí utilizan las ejecutorias de que proviene dicha tesis al definir ese subprincipio en el sentido de que la medida interviniente en un derecho fundamental "debe ser razonable, de tal forma que cuanto más intenso sea el límite de la garantía individual, mayor debe ser el peso o jerarquía de las razones constitucionales que justifiquen dicha intervención". 52 Por otra parte, aunque no de manera palmaria como la Segunda Sala, también dicha tesis habla de la indispensable superación concurrente de los subprincipios de idoneidad, necesidad y proporcionalidad stricto sensu; eso se desprende de una elemental interpretación gramatical de la tesis (también éstas tienen que ser objeto de labor hermenéutica): la SCJN habla de que la medida legislativa en cuestión "debe" superar los parámetros contenidos en sus incisos $a, b, c$ y $d$; el sentido de tal conjunción es indiscutible y da a entender que no habla de uno o alguno de esos elementos, sino de todos y cada uno de ellos.

Acción de inconstitucionalidad 14/2004 (voto particular del ministro Góngora Pimentel), Diario Oficial de la Federación, 4 de octubre de 2004, 2a. sección, pp. 120-122.

51 Véase supra, notas 1 y 2.

52 Op. cit., nota 46, p. 834. Véase también “TRANSPARENCIA Y ACCESO A LA INFORMACIÓN PÚBLICA GUBERNAMENTAL. EL ARTÍCULO 14, FRACCIÓN I, DE LA LEY FEDERAL RELATIVA, NO VIOLA LA GARANTÍA DE ACCESO A LA INFORMACIÓN", tesis 2a. XLIII/ 2008, Semanario Judicial de la Federación y su Gaceta, Novena Época, t. XXVII, abril de 2008, p. 733. 
La jurisprudencia 130/2007 no dice con todas sus letras, pero sí las ejecutorias correspondientes, que la base constitucional textual del principio de proporcionalidad es el artículo 16 constitucional, concretamente su prohibición de arbitrariedad que se relaciona con la motivación material exigida por ese precepto. ${ }^{53}$ Esta disposición contiene, según una de las pocas opiniones jurídicas firmemente establecidas en México, "el principio de legalidad [que] se extiende también al Poder Legislativo, ya que éste se encuentra sujeto a normas de rango constitucional"; ${ }^{4}$ así, esta importantísima disposición constitucional en nuestro país hace el principio de proporcionalidad taxativamente aplicable en todo caso, según su intérprete definitivo; lo anterior no es nada irrelevante: disipa muchas dudas sobre la aplicabilidad del examen de proporcionalidad y la manera en que la Constitución ordena su empleo. Si bien admitimos que no es fácil sintetizar todas las bases teóricas del principio de proporcionalidad en términos llamados a una vida práctica inmediata, ${ }^{55}$ creemos que habría sido muy conveniente que se estableciera con mayor precisión y claridad en las ejecutorias correspondientes y la tesis jurisprudencial comentada, que también lo apoyan la óptima eficacia de los derechos fundamentales que manda el artículo 1o. constitucional, relacionado con el principio pro homine que establece la Convención Americana de Derechos Humanos y la unidad del ordenamiento constitucional; pues todos estos elementos influyeron de distintas maneras en dichos actos jurisdiccionales. ${ }^{56}$

53 Op. cit., nota 46, p. 831; y Sánchez Gil, op. cit., nota 48, pp. 63 y ss. Señalando que ese precepto constitucional impone "límites de razonabilidad" al legislador, véase “AHORRO Y CRÉDITO POPULAR. EL ARTÍCULO CUARTO TRANSITORIO DEL DECRETO POR EL QUE SE REFORMAN Y ADICIONAN DIVERSAS DISPOSICIONES DE LA LEY RELATIVA, PUBLICADO EN EL DIARIO OFICIAL DE LA FEDERACIÓN EL 27 DE MAYO DE 2005, NO VIOLA EL PRINCIPIO DE LEGALIDAD”, tesis 1a. CCXXIX/2007, Semanario Judicial de la Federación y su Gaceta, Novena Época, t. XXVI, octubre de 2007, p. 181.

54 Pleno, acción de inconstitucionalidad 36/2001, Diario Oficial de la Federación, 13 de mayo de 2003, 1a. sección, con. V, p. 60.

55 Véase Sánchez Gil, op. cit., nota 48, pp. 23 y ss.

56 Op. cit., nota 46, pp. 821-822 y 828-829. Véase "IGUALDAD. CASOS EN LOS QUE EL JUEZ...", cit., nota 6. 


\section{PeRsPeCtivas DE APLICACIÓN}

Los usos que puede tener la jurisprudencia 130/2007 son múltiples y no podrían ser relacionados ex ante dentro de un catálogo cerrado, especialmente por su anclaje textual en el artículo 16 de la Constitución. Pero indudablemente los más relevantes serán del ámbito de la ley fundamental.

Para la interpretación constitucional resultará una importantísima herramienta. La aplicación sin reparos del principio de proporcionalidad en ella deberá de conducir a una verdadera hermenéutica de la ley fundamental: una actividad interpretativa que posea matices, precisión y busque la justicia en sentido no sólo formal sino también material; ${ }^{57}$ una lectura de la Constitución que no sea un burdo tijeretazo sino una incisión quirúrgica.

Un punto importante es la indispensable profundización en el bagaje teórico y sobre todo axiológico que representa el principio de proporcionalidad. Difícilmente habrá una correcta aplicación de la jurisprudencia que nos ocupa, tanto de un lado como del otro de la barandilla, si sus fundamentos - los mismos del Estado constitucional democrático-no son conocidos en suficiente medida para que se deriven sus implicaciones y puedan ser correctamente aplicados. El principio de proporcionalidad, finalmente, sólo suministra algunos criterios que en último grado toca al operador jurisdiccional llevar a efecto, y para eso tiene que conocer de qué trata ese concepto y su significado para el orden jurídico.

El examen de proporcionalidad tiene en México una parcela de singular relevancia: el efectivo control constitucional de las atribuciones legislativas. La razonabilidad jurídica lleva a que el legislador haya de esforzarse por ofrecer verdaderas justificaciones para sus actos, evitando la imposición arbitraria de medidas incidentes en el goce de los derechos de los ciudadanos, aun cuando el propio Constituyente le autorice a regularlos; una férrea aplicación del principio de proporcionalidad obligará al legislador a tomar en serio la Constitución y los derechos fundamentales..$^{58}$ Es increíble la ligereza con que los órganos legislativos

57 Véase Beuchot, Mauricio, Hermenéutica analógica y filosofia del derecho, San Luis Potosí, UASLP, Facultad de Derecho, 2007, pp. 129 y 142-146.

58 Como sugirió la Primera Sala en reciente criterio: "RESTRICCIONES A LOS DERECHOS FUNDAMENTALES. ELEMENTOS QUE EL JUEZ CONSTITUCIONAL DEBE TOMAR EN CUENTA PARA CONSIDERARLAS VÁLIDAS", tesis 1a. LXVI/2008, Semanario Judicial de la Federación y su Gaceta, Novena Época, t. XXVIII, julio de 2008, p. 462. 
pretenden justificar las normas generales que expiden, a la cual dan visto bueno los juzgadores; por no entrar éstos a un examen mucho más profundo al relativo a constatar que en el proceso legislativo se dijo que una ley determinada cumple tales y cuales fines, en las sentencias de control constitucional se tienen por justificadas muchas vulneraciones a derechos fundamentales "apoyadas" por los motivos más baladíes, sin que en realidad tiendan de manera imprescindible a la satisfacción de un fin legítimo. En algo se ha avanzado últimamente al reconocerse la obligación de las legislaturas, de ofrecer una "motivación reforzada" a algunos de sus actos; 59 pero de nada sirve una mera expresión formal de objetivos legislativos, si los tribunales no analizan detenidamente la "relación de instrumentalidad" 60 entre éstos y la medida a que supuestamente sirven, o sea la de "proporcionalidad entre los medios y los fines que [ésta] pretende alcanzar", ${ }^{61}$ porque significaría un aval a decisiones autoritarias del legislador que contravienen el contenido esencial de la Constitución.

59 "FINES EXTRAFISCALES. CORRESPONDE AL ÓRGANO LEGISLATIVO JUSTIFICARLOS EXPRESAMENTE EN EL PROCESO DE CREACIÓN DE LAS CONTRIBUCIONES", tesis 1a./J. 46/2005, Semanario Judicial de la Federación y su Gaceta, novena época, t. XXI, mayo de 2005, p. 157; "PENAS Y SISTEMA PARA SU APLICACIÓN. CORRESPONDE AL PODER LEGISLATIVO JUSTIFICAR EN TODOS LOS CASOS Y EN FORMA EXPRESA, LAS RAZONES DE SU ESTABLECIMIENTO EN LA LEY", tesis 1a. LXIX/2006, Semanario Judicial de la Federación y $s u$ Gaceta, novena época, t. XXIII, abril de 2006, p. 158; "EXENCIONES TRIBUTARIAS. LAS RAZONES PARA JUSTIFICARLAS DEBEN ADVERTIRSE CLARAMENTE DE LA LEY O EXPRESARSE EN EL PROCESO LEGISLATIVO EN QUE SE SUSTENTAN",tesis 2a./J. 70/2006, Semanario Judicial de la Federación y su Gaceta, novena época, t. XXIV, julio de 2006, p. 353; y "COlegios DE PROFESIONISTAS. El ARTículo 44 DE LA LEY REglamentARIA DEL ARTículo 5o. CONSTITUCIONAL, RELATIVO AL EJERCICIO DE LAS PROFESIONES EN EL DISTRITO FEDERAL, AL LIMITAR A CINCO EL NÚMERO MÁXIMO DE COLEGIOS SUSCEPTIBLES DE CONSTITUIRSE POR CADA RAMA, VULNERA LA GARANTÍA DE IGUALDAD, EN RELACIÓN CON LAS DE LIBERTAD DE TRABAJO Y ASOCIACIÓN", tesis 1a. CCXXXVII/2007, Semanario Judicial de la Federación y su Gaceta, novena época, t. XXVI, octubre de 2007, p. 184. Véase también “EQUIDAD TRIBUTARIA. LA OMISIÓN DEL ÓRGANO LEGISLATIVO DE JUSTIFICAR LAS RAZONES QUE SUSTENTAN UN TRATO DIFERENCIADO EN EL PROPIO PROCESO DE REFORMAS A UN ORDENAMIENTO LEGAL, POR Sí MISMA, NO CONLLEVA LA INCONSTITUCIONALIDAD DE LA NORMA", tesis 1a. CLXXIX/2007, Semanario Judicial de la Federación y su Gaceta, novena época, t. XXVI, septiembre de 2007, p. 384.

60 Supra, nota 33.

61 Op. cit., nota 46, p. 831. 
En general una de las más notables aplicaciones del principio de proporcionalidad se ha dado en el campo de la moderación de las penas, pero últimamente en él jugó un importante papel la jurisprudencia 130/2007 que comentamos. Basándose expresamente en este precedente, en la acción de inconstitucionalidad 31/2006 que resolvió el 19 de febrero de 2008, el Pleno sentó con meridiana claridad que el legislador penal no debe establecer penas cuya gravedad supere la del delito del que deriven o que por algún otro motivo carezcan de "razonabilidad", ${ }^{62}$ y en esta resolución vemos cuatro aspectos poderosamente llamativos. ${ }^{63} \mathrm{El}$ primero de ellos es que con dicho criterio se afianza la aplicabilidad del examen de "proporcionalidad de las penas", ${ }^{64}$ y no es que la idea fuera nueva, sino que la estableció la ya citada tesis aislada de la Primera Sala ${ }^{65}$ y no una jurisprudencia plenaria como ahora; el segundo, que la misma decisión da claro contenido a ese poco entendido e indeterminado concepto: dicha proporcionalidad se establece al examinar la idoneidad, necesidad y ponderación de la medida dictada por el legislador cuya libertad de configuración, aunque amplia, no es ilimitada porque "debe respetar el contenido de diversos principios constitucionales, dentro de los cuales se encuentra el de proporcionalidad y razonabilidad jurídica" que define la jurisprudencia 130/2007 invocada en esa opinión; en tercer lugar, obliga al juez constitucional a analizar si en las leyes penales "exist[e] proporción y razonabilidad suficiente entre la cuantía de la pena y la gravedad del delito", lo que aunado a la suplencia de la queja en el juicio de amparo, implica reconocer amplísimas facultades a todos los juzgadores de control constitucional para el minucioso escrutinio de las leyes penales; y finalmente, su obligada trascendencia a otros ámbitos como el derecho administrativo sancionador, ${ }^{66}$ y por los alcances que la SCJN admite al

62 El estudio de este tema excede por supuesto los límites de este trabajo, pero no dejamos de recomendar la lectura de la ejecutoria que referimos junto con la de Islas de González Mariscal, Olga, y Carbonell, Miguel, El artículo 22 constitucional y las penas en el Estado de derecho, México, UNAM, Instituto de Investigaciones Jurídicas, 2007.

63 Semanario Judicial de la Federación y su Gaceta, novena época, junio de 2008, t. XXVII, p. 675.

64 Muy al margen de la reforma constitucional publicada el 18 de junio de 2008, que "nada más" explicita la faceta penal sustantiva del principio que estudiamos y ya contenía el primer párrafo artículo 16 de la ley fundamental.

65 "PRISIÓN VITALICIA...", op. cit., nota 28; y "PENAS...", op. cit., nota 59.

66 "DERECHO ADMINISTRATIVO SANCIONADOR. PARA LA CONSTRUCCIÓN DE SUS PROPIOS PRINCIPIOS CONSTITUCIONALES ES VÁLIDO ACUDIR DE MANERA PRUDENTE A 
artículo 22 constitucional en general para cualquier sanción jurídica sin importar la materia en que se hubiera establecido, como se indicó en la contradicción de tesis 21/2006-PL.67

Sin duda y a pesar de su sencillez en abstracto, el juicio de proporcionalidad puede dar dificultades en la práctica, especialmente cuando se trata de mantener el respeto judicial a la libertad de configuración del legislador; ${ }^{68}$ su correcta aplicación no es un juego de niños, y requiere una buena dosis de sutileza del operador. Si bien dicho principio lleva a un control efectivo y más amplio sobre el Poder Legislativo, también los juzgadores deben actuar "proporcionadamente" para hallar el punto medio en el que consistirá la justicia del caso, y respetar los ámbitos de actuación de las autoridades justiciables. No obstante, los eventuales problemas de este principio no son nada, comparados a la total ausencia de criterios objetivos de "razonabilidad"; en todo caso, exigirán de los juzgadores y postulantes un nivel más elevado de argumentación que habrán de estar preparados para dar, y esto es considerablemente preferible a una aplicación subjetiva y caprichosa del derecho, máxime tratándose del constitucional.

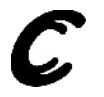

LAS TÉCNICAS GARANTISTAS DEL DERECHO PENAL, EN TANTO AMBOS SON MANIFESTACIONES DE LA POTESTAD PUNITIVA DEL ESTADO", tesis P./J. 99/2006, Semanario Judicial de la Federación y su Gaceta, novena época, t. XXIV, agosto de 2006, p. 1565.

67 Al aplicar el subprincipio de necesidad: "PATRIA POTESTAD. EL SUPUESTO NORMATIVO QUE IMPONE SU PÉRDIDA POR ABANDONO INJUSTIFICADO DEL HOGAR CONYUGAL POR MÁS DE 6 MESES, VIOLA EL ARTíCULO 4o. DE LA CONSTITUCión POLÍTICA DE Los Estados Unidos MeXICANOS", tesis P./J. 62/2008, Semanario Judicial de la Federación y su Gaceta, Novena Época, junio de 2008, t. XXVII, p. 8.

68 Véase "ANÁliSIS CONSTITUCIONAL. SU INTENSIDAD A LA LUZ DE LOS PRINCIPIOS DEMOCRÁTICO Y DE DIVISIÓN DE PODERES”, tesis 1a./J. 84/2006, Semanario Judicial de la Federación y su Gaceta, novena época, noviembre de 2006, t. XXIV, p. 29. 